Gefässchirurgie 2015 · 20:214-216

DOI 10.1007/s00772-015-0009-6

Online publiziert: 24. März 2015

(c) Springer-Verlag Berlin Heidelberg 2015

\section{U. Kusenack ${ }^{1} \cdot$ P. Haage ${ }^{2} \cdot$ A. Terzis $^{3} \cdot$ K. Meletiadis $^{1}$}

${ }^{1}$ Klinik für Gefäßchirurgie, Helios Klinikum Wuppertal, Klinikum der Universität Witten-Herdecke, Wuppertal

2 Zentrum für Radiologie, Helios Klinikum Wuppertal, Klinikum der Universität Witten-Herdecke, Wuppertal

${ }^{3}$ Klinik für Neurochirurgie, Helios Klinikum Wuppertal, Klinikum der Universität Witten-Herdecke, Wuppertal

\title{
Aortokavale Fistel mit kardialer Dekompensation als Spätkomplikation nach Aortenstenting
}

\section{Anamnese}

Ein 86-jähriger männlicher Patient stellte sich mit sehr starken, akut aufgetretenen Schmerzen im rechten Bein und in der rechten Flanke sowie rapider Verschlechterung des Allgemeinzustands in unserem Notfallzentrum vor. Drei Jahre zuvor war ihm aufgrund eines infrarenalen Bauchaortenaneurysmas ein aortobiiliakaler Stentgraft implantiert worden.

\section{Klinischer Befund und Diagnostik}

Herausragende Pathologie zum Aufnahmezeitpunkt war ein akutes Nierenversagen mit einem Serumkreatinin von $6,4 \mathrm{mg} / \mathrm{dl}$ und ein Kalium-Wert von $6,2 \mathrm{mmol} / \mathrm{l}$. Im transthorakalen Echokardiogramm (TTE) bestanden deutliche Anzeichen einer akuten Rechtsherzbelastung.

Eine notfallmäßig durchgeführte kontrastmittelgestützte Computertomographie (CT) von Thorax und Abdomen zeigte ein Endoleak Typ I am distalen Ende des rechten iliakalen Prothesenschenkels (• Abb. 1). Ursache des Typ-I-Endoleaks war am ehesten ein in den Aneurysmasack retrahierter und abgeknickter iliakaler Prothesenschenkel.

Überraschenderweise war es an gleicher Stelle zur Ausbildung einer aortokavalen Fistel mit konsekutiver Rechtsherzbelastung - erkennbar an einer Leber- und Nierenstauung - gekommen. Zudem komprimierte das reperfundierte
Aortenaneurysma die infrarenale V. cava deutlich (• Abb. 2).

- Abbildung 3 zeigt das ursprüngliche postoperative Ergebnis nach endovaskulärer Aneurysmaausschaltung (EVAR) 2009 mit sehr kurzer Landezone rechtsiliakal.

\section{Therapie und Verlauf}

Unter der Arbeitsdiagnose „dekompensierte aortokavale Fistel aufgrund eines Typ-Ib-Endoleaks nach EVAR“ implantierten wir noch am Aufnahmetag in Intubationsnarkose einen iliakalen Prothesenschenkel rechts zur Verlängerung der Beckenachse und damit zur Abdichtung des Endoleaks bis in die A. iliaca externa (bei verschlossener A. iliaca interna).

Gleichzeitig kam es durch diese Prozedur zu einem sofortigen und klinisch wirksamen Verschluss der aortokavalen Fistel.

Die Nierenretentionswerte normalisierten sich trotz Kontrastmittelapplikation zügig (Kreatinin 1,4 mg/dl am vierten postoperativen Tag), sodass eine Hämofiltration nicht indiziert war.

Das TTE zeigte nun eine rekompensierte, nahezu normale Pumpfunktion ohne Nachweis einer weiteren Rechtsherzbelastung.

Aufgrund der weiterhin deutlichen Kompression der V. cava inferior durch das ausgeschaltete Aneurysma entschlossen wir uns, den Patienten zur Prophylaxe einer Kavathrombose für 3 Monate mit Rivaroxaban antikoagulatorisch zu behandeln und verlegten den Patienten in eine Anschlussheilbehandlung.

Unmittelbar vor dortiger Entlassung entwickelte der Patienten einen Infekt im Bereich eines vorbestehenden subkutanen Hämatoms in der linken Leiste. Dies führte zur erneuten stationären Aufnahme in unsere Klinik mit operativer Ausräumung des infizierten Hämatoms und systemischer Gabe eines Breitbandantibiotikums.

Am zweiten postoperativen Tag klagte der Patient plötzlich über akute Kopfschmerzen und entwickelte eine Anisoko-

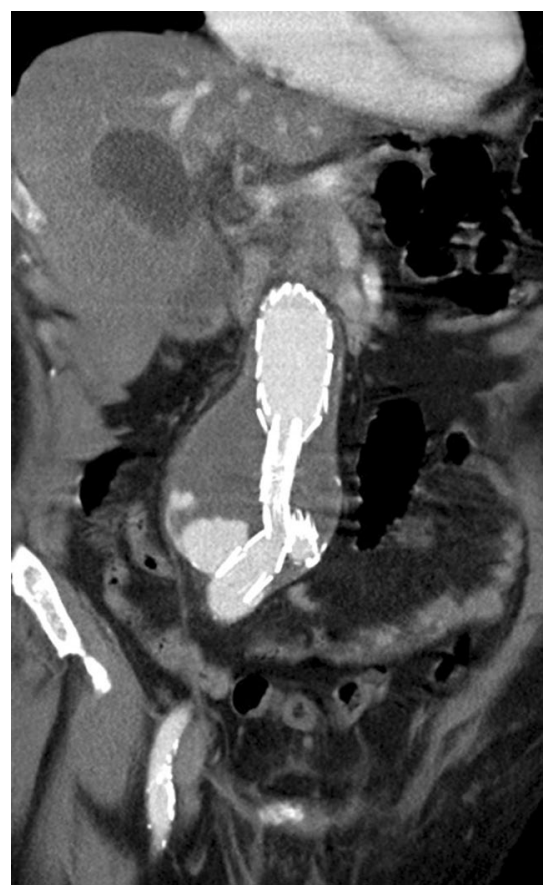

Abb. $1 \Delta$ Retrahierter und abgeknickter Prothesenschenkel iliakal rechts mit Endoleak Typ Ib 


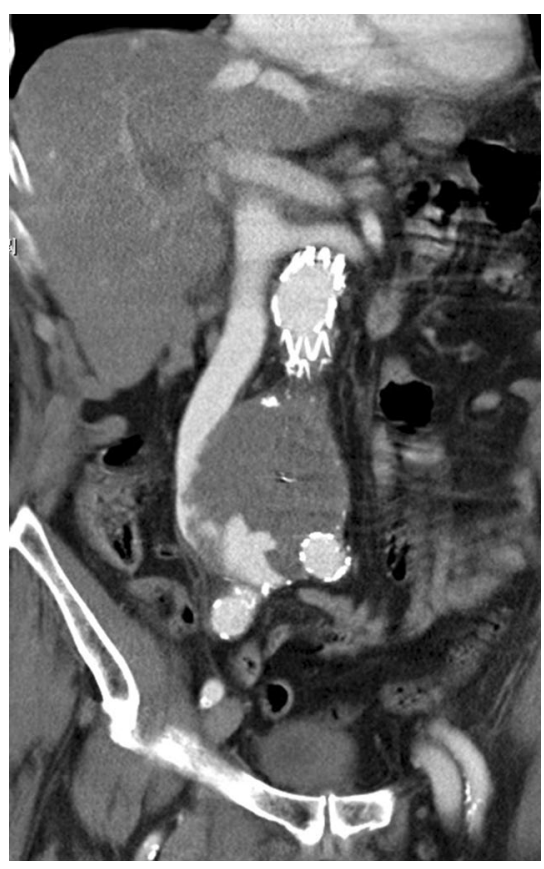

Abb. $2 \Delta$ Ausbildung einer aortokavalen Fistel im Rahmen des Endoleaks Typ Ib

rie links mit Hemiparese rechts sowie eine zunehmende Bewusstseinstrübung.

Das notfallmäßig durchgeführte Schädel-CT ergab als Ursache ein ausgedehntes subdurales frisches Hämatom links auf dem Boden eines älteren Hämatoms mit Mittellinienverlagerung (•Abb.4).

Dies erforderte eine sofortige neurochirurgische Bohrloch-Trepanation mit einer Revision am Folgetag aufgrund einer weiteren Zunahme des subduralen Hämatoms. Trotz erneuter Ausräumung des Hämatoms konnte keine klinische Verbesserung des Patienten (tief komatös, Strecksynergien auf Schmerzreiz) erzielt werden und der Patient verstarb schließlich nach Einstellen der Therapie bei infauster Prognose am 7. postoperativen Tag.

Im Gespräch mit den Angehörigen konnte retrospektiv ein mehrere Wochen zurückliegendes Schädel-Hirn-Trauma (Stoß gegen einen Schrank) eruiert werden.

\section{Diskussion}

Ein distales Endoleak Typ I nach Implantation eines Aortenstentgrafts kann durch korrekte Prothesenplanung im Vorfeld in der Regel vermieden werden [1].

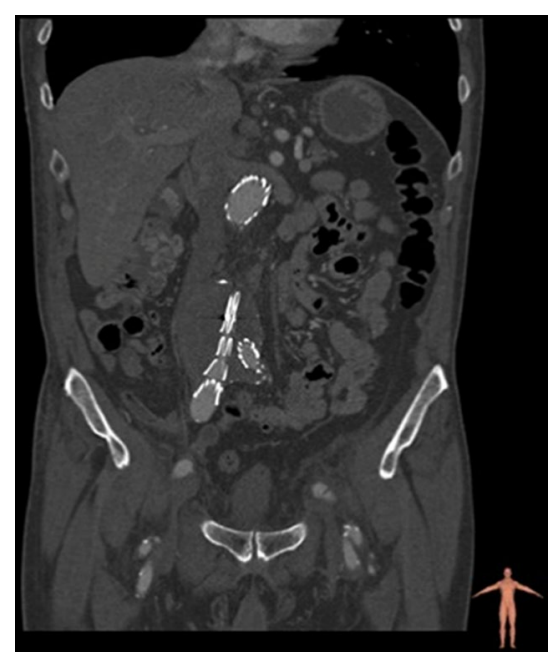

Abb. 3 A Primärergebnis nach EVAR 2009 mit zu kurzer iliakaler Landezone rechts

Ursächlich für das Endoleak war in unserem Falle sicherlich ein - im Rahmen des Primäreingriffes 2009 - zu kurz gewählter iliakaler Prothesenschenkel rechts. Zudem unterblieben weitere engmaschige Nachkontrollen des Aneurysmas mittels Sonographie oder CT. Dies führte im Verlauf von knapp 3 Jahren auch unter Fortschreiten der Dilatation in der A. iliaca communis - zu einem $\mathrm{Zu}$ rückrutschen des rechten iliakalen Prothesenschenkels in den Aneurysmasack mit konsekutiver Entstehung eines TypIb-Endoleaks.

Vermutlich förderte die mechanische Irritation an der Gefäßinnenwand durch das distale Stentende die Entwicklung einer pathologischen Kurzschlussverbindung zwischen Aortengabel und distaler V. cava inferior mit allen Folgen einer akuten Rechtsherzbelastung.

Die Entstehung einer aortokavalen Fistel nach EVAR ist unseres Wissens nach in der Literatur bisher nicht beschrieben worden. Beschrieben ist allerdings die Abdichtung aortokavaler Fisteln im Rahmen gedeckt rupturierter Aortenaneurymen durch einen Stentgraft [2-5].

Eine vergleichsweise einfache operative Maßnahme (Stentgraftverlängerung iliakal rechts) konnte das Endoleak und die aortokavale Fistel mit Rechtsherzinsuffizienz und allen Begleiterscheinungen sofort und dauerhaft beseitigen.

Unser Fall zeigt deutlich, wie wichtig eine ausreichende Landezone für den

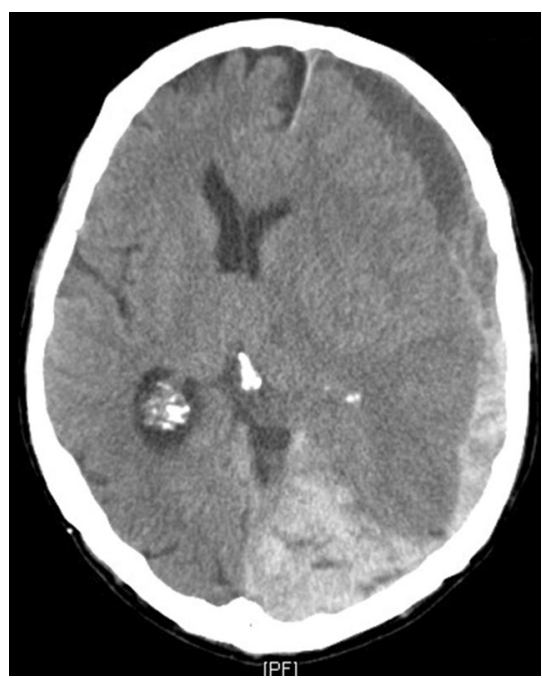

Abb. $4 \Delta$ Schwere intrakranielle Blutung mit Mittellinienverlagerung

Stentgraft auch nach distal und wie notwendig eine regelmäßige Überwachung zur frühzeitigen Detektion eines Endoleaks - insbesondere bei kurzer iliakaler Landezone - ist.

Die prophylaktische Gabe eines Antikoagulans (hier Rivaroxaban) zur Prophylaxe einer Kavathrombose war retrospektiv übertherapiert und sogar gefährlich - wie der weitere Verlauf leider zeigte. Letztlich muss davon ausgegangen werden, dass die Gabe des Antikoagulans zur tödlichen Hirnblutung führte, auch wenn den Antikoagulanzien der neueren Generation eine signifikante Reduktion schwerer, insbesondere intrakranieller Blutungsereignisse nachgesagt wird [6]. Wie lange das subdurale Hämatom bereits bestand, ist unklar. Wahrscheinlich war es im Rahmen des mehrere Wochen zurückliegenden Traumas entstanden. Unter der Gabe von Rivaroxaban kam es zur zweizeitigen Blutung mit tödlichem Ausgang. Leider war das Schädeltrauma anamnestisch nicht eruiert worden.

\section{Fazit für die Praxis}

- Neben der proximalen Landezone ist auch eine ausreichende distale „sealing-zone" zur Vorbeugung eines Endoleaks nach EVAR ein zentraler Aspekt. Andernfalls kann es - insbesondere unter Fortschreiten der dilatativen Grunderkrankung - zur Ausbildung eines distalen Endoleaks Typ I 
mit konsekutiver aortokavaler Fistel und Rechtsherzbelastung kommen.

- Patienten mit kritischem Primärergebnis nach EVAR müssen daher engmaschig bildgebend nachkontrolliert werden.

- Die prophylaktische Gabe eines Antikoagulans zur Verhinderung einer Kavathrombose kann letale Folgen haben und muss daher streng gestellt und anamnestisch gut abgesichert werden.

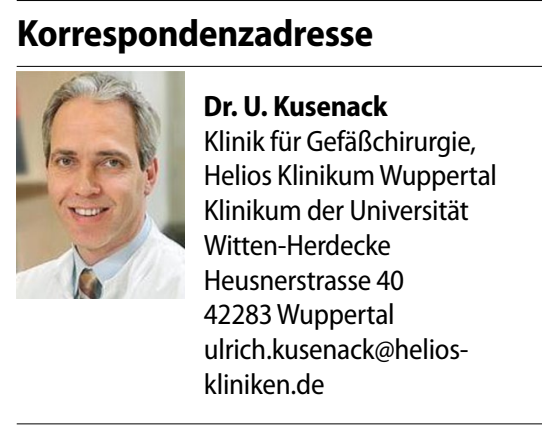

Einhaltung ethischer Richtlinien

Interessenkonflikt. U. Kusenack, P. Haage, A. Terzis und K. Meletiadis geben an, dass kein Interessenkonflikt besteht.

Dieser Beitrag beinhaltet keine Studien an Menschen oder Tieren.

\section{Literatur}

1. Ferrari M, Berchiolli R, Sardella SG, Cioni R, Petruzzi P, Del Corso A, Di Mitri R, Croce C, Romagnani F, Adami D, Mosca F (2005) Endovascular repair of an aorto-left renal vein fistula due to a ruptured abdominal aortic aneurysm after EVAR. J Endovasc Ther 12:512-515

2. Adili F, Balzer JO, Ritter RG, Schmandra TC, Tenholt M, VogI TJ, Schmitz-Rixen T (2004) Ruptured abdominal aortic aneurysm with aorto-caval fistula. J Vasc Surg 40:582

3. Jeuriens-van de Ven $S A$, Schouten van der Velden AP, Schultze Kool LJ, van der Vliet JA, Berger $P$ (2011) Persisting iliaco-caval fistula after EVAR maintained by a type II endoleak. Ann Vasc Surg 25(1142):e1147-1149

4. Melas N, Saratzis A, Saratzis N, Lazaridis I, Kiskinis D (2011) Inferior vena cava stent-graft placement to treat endoleak associated with an aortocaval fistula. J Vasc Surg 18:250-254

5. Siepe M, Koeppe S, Euringer W, Schlensak C (2009) Aorto-caval fistula from acute rupture of an abdominal aortic aneurysm treated with a hybrid approach. J Vasc Surg 49:1574-1576

6. Stoberock K, Debus ES (2013) Neue orale Antikoagulanzien. Gefässchirurgie 18:665-674

\title{
Hier steht eine Anzeige.
}

\author{
Springer
}

